\title{
Medición del impacto económico de las terminales portuarias del Callao: Un análisis mediante la Matriz Insumo - Producto
}

\author{
ELVIS APARCo ${ }^{a}$, VÍCTOR CHANG ${ }^{\text {b }}$ \\ a Universidad Nacional Mayor de San Marcos, Facultad de Ciencias Económicas, Calle Germán \\ Amézaga, $n^{\circ}$ 375, Ciudad Universitaria, C.P. 15081, Perú. E-mail: elvis.aparco@unmsm.edu.pe \\ $b$ Universidad Ricardo Palma, Facultad de Ciencias Económicas y Empresariales, Av. Benavides, $n^{\circ}$ \\ 5440, Santiago de Surco, C.P. 15039, Perú. E-mail: victor.chang@urp.edu.pe
}

\begin{abstract}
RESUMEN
El artículo tiene como objetivo medir el impacto de la inversión y los gastos en operación de las terminales portuarias del Callao en el 2014. Para tal fin, se emplea la Matriz Insumo - Producto de 14 sectores económicos del Perú. Los resultados muestran un impacto de USD 598 millones en el valor agregado, la generación de 66 mil empleos, y que el gasto en operación contribuye más al valor agregado y la generación de empleo que la inversión. Asimismo, se evidencia que la Terminal Muelle Norte presentó un mayor impacto total y la Terminal de Concentrados de Minerales mayor impacto marginal.
\end{abstract}

Palabras clave: Matriz Insumo Producto, Impacto económico portuario, Puerto del Callao (Perú), Inversiones, Operaciones.

\section{Measuring the Economic Impact in Port Terminals of Callao: An Analysis using Input - Output Table}

\begin{abstract}
The objective of this paper is to measure the impact of the investment and operating expenditure (OPEX) of the Callao port terminals in 2014. For this purpose, the input-output table of 14 economic sectors of Peru is used. The results show an impact of USD 598 million in value added, the generation of 66 thousand jobs, and that operating expenditure contribute more to added value and employment creation than investment. Furthermore, it highlights that the North Terminal had a greater total impact and the Mineral Concentrates Terminal had greater marginal impact.

Keywords: Input-output table, port economic impact, Port of Callao (Peru), investment, operation.
\end{abstract}

Clasificación JEL: H54, R42, D57

Artículo recibido en julio de 2018 y aceptado en septiembre de 2018

Artículo disponible en versión electrónica en la página www.revista-eea.net, ref. ə-36313 


\section{INTRODUCCIÓN}

Los puertos constituyen un medio importante para el desarrollo de los países al ser el principal medio de transporte para realizar el comercio. Estos presentan mayores ventajas en comparación con otras alternativas intermodales como el transporte aéreo, por carreteras o vías férreas respecto al flete (Musso, Benacchio \& Ferrari, 2000). De acuerdo con la UNCTAD (2015), cerca del $80 \%$ del comercio mundial se moviliza por vía marítima. En ese sentido, se resalta la importancia de la infraestructura portuaria como soporte de la industria del transporte marítimo al contribuir en la mejora de la accesibilidad, competitividad y, en general, la estructura industrial de un país o región donde opera el puerto (Acciaro, 2008).

En el caso peruano, dada la extensa costa con la que cuenta el Perú, que abarca $2250 \mathrm{Km}$, el puerto del Callao cumple un rol fundamental en la economía peruana al movilizar la mayor cantidad de carga en comparación con otros puertos peruanos de uso público, como el puerto de Pisco, Matarani, Salaverry o Paita. Al respecto, en el 2017 el puerto del Callao movilizó el 88\% de TEU's en el país (Organismo Supervisor de la Inversión en Infraestructura de Transporte de Uso Público - Ositran, 2018), y fue el sexto puerto más importante en América Latina y el Caribe, de acuerdo con el ranking portuario del 2016, elaborado por Cepal (2018) ${ }^{1}$.

Tabla 1

Ranking de TEU's movilizados en puertos de América Latina y el Caribe.

\begin{tabular}{|l|l|c|}
\hline \multicolumn{1}{|c|}{ Puerto } & \multicolumn{1}{c|}{ País } & Miles deTEU's \\
\hline Santos & Brasil & 3394 \\
\hline Colón & Panamá & 3258 \\
\hline Balboa & Panamá & 2990 \\
\hline Manzanillo & México & 2581 \\
\hline Cartagena & Colombia & 2301 \\
\hline Callao & Perú & 2055 \\
\hline Guayaquil & Ecuador & 1822 \\
\hline Kingston & Jamaica & 1567 \\
\hline Buenos Aires & Argentina & 1352 \\
\hline
\end{tabular}

Fuente: Cepal (2018).Elaboración propia.

Dada la importancia de los puertos en la economía peruana, en los últimos años las políticas del sector se han orientado a fomentar mayor inversión y mejorar la gestión de la infraestructura portuaria mediante la conformación de Asociaciones Público - Privadas (APP) con el fin de mejorar la eficiencia y la

${ }^{1}$ Según el "Ranking de puertos. Los Top 20 en América Latina y el Caribe en 2016” elaborado por la Cepal. Disponible en http://perfil.cepal.org/l/es/portmovements_classic.html [Último acceso: 01/04/2018] 
productividad de los puertos. Si bien se ha evidenciado que estas reformas han mejorado el desempeño de los puertos peruanos (Chang \& Tovar, 2014a,b; 2017a,b), no existe evidencia empírica sobre el impacto económico que han generado en la economía peruana. En particular, las reformas en el puerto del Callao han permitido realizar mayores inversiones en tres terminales portuarias, la terminal de contenedores Muelle sur, la terminal multipropósito Muelle Norte y la terminal de embarque de concentrado de minerales, así como mejorar la gestión de la infraestructura portuaria; sin embargo, a la fecha no se ha realizado una evaluación sobre el impacto que ha generado la modernización del puerto en términos de valor agregado y empleo en la economía peruana.

De esta manera, el objetivo principal de este estudio es medir el impacto económico del gasto destinado a la operación e inversiones ejecutadas en las terminales portuarias del Callao para el año 2014. La metodología que se emplea consiste en un modelo basado en la Matriz Insumo - Producto (MIP), donde los gastos e inversiones ejecutadas en el puerto generan variaciones en el valor agregado y el empleo. Asimismo, se miden los impactos de forma sectorial y disgregada en impactos directos, indirectos e inducidos.

A nivel temático y metodológico, en relación con otros estudios empíricos, el presente artículo es el primero que aborda los impactos generados de los gastos destinados a la operación del puerto y las inversiones ejecutadas en infraestructura portuaria en el Perú utilizando la MIP.

La estructura del presente artículo es la siguiente, posterior a la introducción, la sección dos ilustra el proceso de reformas portuarias en el puerto del Callao. La sección tres abarca una revisión de la literatura relevante, en tanto la sección cuatro muestra la metodología empleada. La sección cinco muestra una descripción de los datos y en la sección seis se muestran los resultados. Por último, en la sección siete se exponen las principales conclusiones y recomendaciones que se derivan de este estudio.

\section{PROCESO DE REFORMAS EN EL PUERTO DEL CALLAO}

Desde 1970, la administración, operación, equipamiento y mantenimiento de las terminales portuarias de uso público en Perú se encontraba en manos de La Empresa Nacional de Puertos del Perú (ENAPU). Sin embargo, debido a los problemas que tenía ENAPU, como la alta dependencia del presupuesto público, excesiva cantidad de trabajadores, la falta de criterios técnicos en la realización de las inversiones, así como el inadecuado esquema tarifario por el uso de la infraestructura, las cuales no eran realizadas sobre la base de los costos de mantenimiento y la insuficiente asignación de recursos financieros que no permitían realizar las inversiones necesarias para la modernización de los puertos; trajeron como consecuencia que el Estado promueva las reformas del sector mediante la conformación de las APPs (Chang \& Tovar, 2014a,b). 
En 1992 mediante el Decreto ley $N^{\circ} 25882$ se incluyó a la ENAPU en el Proceso de Promoción de la Inversión Privada de las Empresas del Estado establecido por el Decreto Legislativo $\mathrm{N}^{\circ} 674$. Al respecto, luego de la concesión del puerto de Matarani, el proceso quedó suspendido en el 2001 (Ley N 270396) debido a la ausencia de un marco legal para el sector portuario peruano. Luego de unos años, tras la promulgación de la Ley Nacional de Puerto (Ley $\mathrm{N}^{\circ}$ 27943) en el 2005 se retoma el proceso de concesión de los principales puertos del país iniciando con la concesión de una terminal nueva exclusiva para la movilización de carga contenerizada en el puerto del Callao.

Desde la administración de ENAPU, el puerto del Callao sólo contaba con la terminal portuaria multipropósito Muelle Norte, la cual presentaba limitaciones en el manejo de carga contenerizada. Principalmente, la falta de profundidad para atender a buques mayores que los Panamax, así como la falta de equipos especializados como grúas pórtico, imposibilitaba que arribaran naves de mayor tamaño y restaba productividad al puerto, al permitir que sólo sea posible el ingreso de buques que contaban con sus propias grúas ${ }^{2}$. Con el fin de atender esta problemática, en el año 2006 el Estado Peruano licitó el diseño, construcción, financiamiento, conservación y explotación de una nueva terminal en el puerto del Callao que movilice exclusivamente carga contenerizada. De esta manera, DP World Callao S.A se adjudicó la Buena Pro de la Terminal Muelle Sur (TMS) al presentar una propuesta de inversiones en obras, equipos e inversión adicional que ascendieron a USD 617 millones $^{3}$. Cabe señalar que, al ser una terminal nueva, ésta recién inició operaciones luego de construido la primera fase en el año 2010. Las inversiones obligatorias correspondientes a dicha fase consistieron en la construcción de dos amarraderos y la adquisición de equipos, entre ellos, seis grúas pórtico.

En tanto, igual de importante era modernizar la antigua terminal multipropósito, la cual comprendía los muelles de carga sólida y líquida a granel, carga de material rodante, carga fraccionada y de contenedores. En 2011 el Estado Peruano suscribió el Contrato de Concesión para el diseño, construcción, financiamiento y explotación de la Terminal Muelle Norte (TMN) del puerto del Callao con el Consorcio APM Terminals S.A por un periodo de 30 años. Ello tras ganar en concurso público en el cual el primer factor de competencia estuvo relacionado con el menor índice tarifario y los dos siguientes con las mayores

\footnotetext{
${ }^{2}$ Para un análisis exhaustivo de la situación del Puerto del Callao en el 2010, remitimos al lector interesado al Plan Maestro de la Terminal portuaria del Callao (Autoridad Portuaria Nacional, 2010).

${ }^{3}$ Cabe mencionar que se dio un empate en el primer factor de competencia, luego de que los tres postores propusieran el menor Índice Tarifario Estándar pedido por el licitante. El segundo factor de competencia, y factor de desempate, fue el de Mayor Inversión Complementaria Adicional.
} 
tasas de descuento. La inversión comprometida para este proyecto ascendió en USD 1069 millones.

Finalmente, ante la alta movilización de carga de minerales por el puerto del Callao y derivado de la contaminación que generaba el manipuleo de este tipo de carga que trajo como consecuencia altos niveles de plomo en la sangre en los niños en las zonas aledañas al puerto (Espinoza et al., 2013); en el 2009 se gestó una iniciativa privada para el embarque de minerales a través de una faja transportadora tubular con sistema de encapsulado. Este tipo de tecnología permitía evitar la contaminación ocasionada por la logística que involucraba la exportación de minerales por el puerto.

En 2011, luego de transcurrido el plazo para la declaratoria de interés de la iniciativa privada a fin de que puedan participar terceros interesados en el proceso de adjudicación, el Estado Peruano concesiona la Terminal Transportadora de Concentrados de Minerales (TCM) a la empresa Transportadora Callao S.A. al no haberse presentado otras empresas interesadas. El Contrato de Concesión abarcaba el diseño, construcción, financiamiento, conservación y explotación de la terminal, así como el realizar una inversión de USD 120 millones.

Figura 1

Mapa del Puerto del Callao

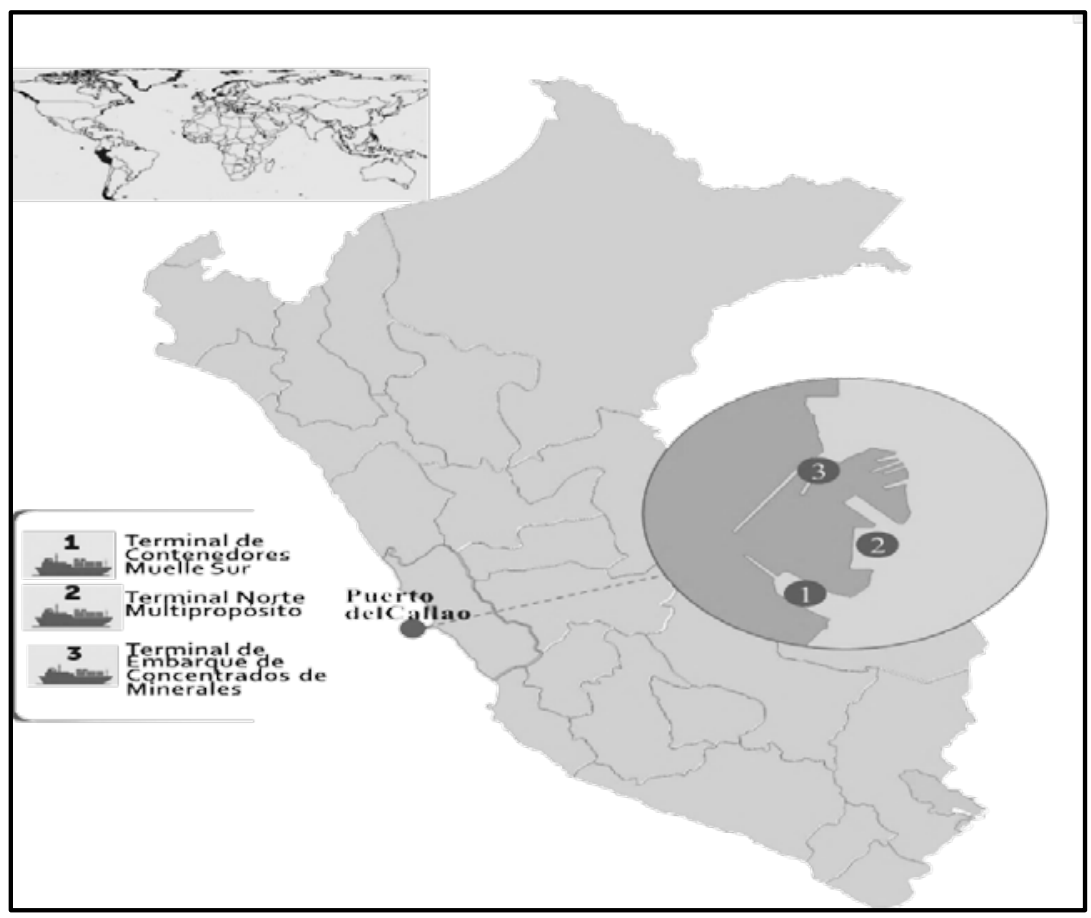

Fuente: Elaboración propia. 
Las inversiones ejecutadas en la TCM concluyeron en 2014 con la construcción de un muelle de $219 \mathrm{~m}$ y un sistema hermético de faja transportadora de concentrados de minerales entre el punto de acceso al público y el muelle. La TCM inició operaciones en 2014 y con ello se concluyó el proceso de reformas en el Puerto del Callao. La Figura 1 muestra las terminales que operan en el Puerto del Callao.

Cabe precisar que todas las terminales fueron concesionadas bajo la modalidad autosostenibles, es decir, que el diseño, construcción y operación del puerto no comprometieron los recursos del estado. Además, los contratos de concesión establecieron que los concesionarios tendrían la exclusividad para operar bajo la modalidad de mono operador, a excepción del servicio de practicaje y remolcaje. Las características de las terminales concesionadas en el puerto del Callao se muestran en la Tabla 2.

Tabla 2

Características del Puerto del Callao, por empresa concesionaria, 2014.

\begin{tabular}{|c|c|c|c|}
\hline & TMN & TMS & TCM \\
\hline \multicolumn{4}{|c|}{ Características del Contrato de Concesión } \\
\hline Año de concesión & 2011 & 2006 & 2011 \\
\hline Tipo de carga & Multipropósito & Contenedores & Minerales \\
\hline Tipo de contrato & Brownfield & Greenfield & Greenfield \\
\hline Compromiso de Inversión (millones de USD) & 884 & 705 & 120 \\
\hline \multicolumn{4}{|c|}{ Características del Tráfico } \\
\hline Naves & 2258 & 840 & 89 \\
\hline Carga (miles de toneladas) & 14895 & 15108 & 1651 \\
\hline Contenedores (miles TEU's) & 491 & 1427 & - \\
\hline Ingresos (millones USD, sin IGV ${ }^{1 /}$ ) & 104,3 & 148,5 & 12,6 \\
\hline \multicolumn{4}{|c|}{ Características de la Infraestructura } \\
\hline Longitud del muelle máximo & $560 \mathrm{~m}$. & $650 \mathrm{~m}$ & $219 \mathrm{~m}$ \\
\hline Amarraderos & 14 & 2 & 1 \\
\hline Dragado máximo & $15,5 \mathrm{~m}$ & $16,0 \mathrm{~m}$ & $13,1 \mathrm{~m}$ \\
\hline
\end{tabular}

1/ IGV: Impuesto General a las Ventas.

Fuente: Ositran (2018), Estados Financieros Auditados y memorias anuales de los concesionarios. Elaboración propia.

\section{REVISIÓN DE LA LITERATURA}

El impacto económico de los puertos puede devenir de las inversiones que en estos se ejecuten, los gastos que se destinen a la operación y mantenimiento, y por las actividades que realizan las industrias conexas a la actividad portuaria. En general, no existen muchos estudios concernientes al impacto de las inversiones en infraestructura portuaria. Entre estos, Park \& Seo (2016) y Song \& Geenhuizen (2014) estiman el impacto de la inversión en capital fijo a través de una función de producción con datos panel en el Producto Bruto Interno (PBI) 
regional para Korea y China, respectivamente. En esa línea, Martínez, Malagón \& Muñoz (2014) utilizan la MIP para estimar el impacto de la ampliación del Puerto de Cartagena en Colombia. Lee, Lee \& Chen, (2012) realizan Modelos de Equilibrio General Computable (MEGC), cuya metodología se basa en representar las principales interrelaciones entre los sectores económicos y agentes económicos.

Por otro lado, los trabajos relacionados a la estimación del impacto derivados de la operación de los puertos pueden clasificarse en dos grupos en relación con las variables que emplean. Artal et al. (2016), Shan, Yu \& Lee (2014) estiman el impacto en el producto a través de variables de tráfico, como el tráfico de mercancías o de pasajeros. Martínez, Malagón \& Muñoz (2014), De Rus, Tovar \& Gonzáles (2009), Braun (1990) utilizan como proxy el gasto destinado a la operación de los puertos.

Asimismo, diversos autores han estudiado el impacto de las actividades conexas a la actividad portuaria. Sobre este punto, las actividades conexas dependerán del tipo de puerto y de sus particularidades; por ejemplo, si bien todos los puertos se relacionan íntimamente con las industrias de líneas navieras, los impactos en puertos de cruceros suelen considerar además las actividades turísticas. Estas se evalúan a través de variables como el tráfico de pasajeros (Artal et al., 2016), gastos de pasajeros y de la tripulación en la zona de influencia (Braun, Xander \& White, 2002) o el desarrollo comercial aledaño al puerto (Chang et al., 2016).

La literatura de medición del impacto económico portuario abarca diversos enfoques metodológicos. En particular, destacan los basados en la Matriz Insumo - Producto. Ello obedece a que este enfoque ofrece resultados bastante detallados en comparación con otros métodos, pudiendo diferenciar entre impactos directos, indirectos e inducidos. Asimismo, permite determinar la importancia del sector portuario respecto a los demás sectores de la economía. En este aspecto, resalta el trabajo de Chang, Shin \& Lee (2014) quien encuentra evidencia a través de los encadenamientos hacia atrás y hacia adelante, que el sector portuario no parece utilizar a otros sectores en su proceso productivo, pero que sí es utilizado exhaustivamente por otros sectores.

Entre los autores que utilizaron la MIP para estimar los impactos de la actividad portuaria resaltan Kwak, Yoo \& Chang (2005) y Chang et al. (2016) quienes estiman el impacto en el valor agregado y el empleo de la industria naviera a nivel nacional y para la ciudad de Icheon, ambos en Corea del Sur. Artal et al. (2016) encuentran que la operación del puerto en Murcia en el transporte de carga y pasajeros en España contribuyó en 1,1\% al PBI y 0,1\% al empleo de la región en el 2011. Braun (1990) estimó a través de los gastos de operación del puerto en el este de Florida contribuyó al PBI regional en USD 
709 millones en 1988. La Tabla 3 muestra un resumen de los autores que utilizan esta metodología.

Tabla 3

Principales estudios relacionados al impacto del sector portuario en la economía en base a la MIP

\begin{tabular}{|c|c|c|c|c|}
\hline Autor & País / Periodo & Puerto & Input & Output \\
\hline $\begin{array}{l}\text { Artal, Gómez, } \\
\text { Navarro \& } \\
\text { Ramos (2016) }\end{array}$ & Murcia, España (2011) & $\begin{array}{l}\text { Puerto de } \\
\text { Cartagena }\end{array}$ & $\begin{array}{l}\rightarrow \text { Tráfico de Mercancías } \\
\rightarrow \text { Tráfico de Pasajeros }\end{array}$ & $\begin{array}{l}\rightarrow 3,81 \% \text { VA de la región. } \\
\rightarrow 2,11 \% \text { PEA de la región. }\end{array}$ \\
\hline $\begin{array}{l}\text { Chang Park, Liu } \\
\text { \& Roh (2016) }\end{array}$ & $\begin{array}{l}\text { Icheon, Corea del Sur } \\
(2016)\end{array}$ & $\begin{array}{l}\text { Puerto de } \\
\text { Icheon }\end{array}$ & $\begin{array}{l}\rightarrow \text { Actividad de Cruceros } \\
\rightarrow \text { Desarrollo Comercial } \\
\text { aledaño al Puerto }\end{array}$ & $\begin{array}{l}\rightarrow \text { USD } 6,4 \text { millones en VA de } \\
\text { Icheon y USD } 47,8 \text { millones en } \\
\text { otras regiones. } \\
\rightarrow 96 \text { empleos en Icheon y } 837 \\
\text { empleos en otras regiones. }\end{array}$ \\
\hline $\begin{array}{l}\text { Danielis \& } \\
\text { Gregori (2013) }\end{array}$ & $\begin{array}{l}\text { Friuli Venecia Giulia, } \\
\text { Italia }\end{array}$ & \begin{tabular}{|l} 
Sistema \\
Portuario de \\
Friuli Venecia \\
Giulia
\end{tabular} & $\begin{array}{l}\rightarrow \text { Interrelación del Sector } \\
\text { Portuario }\end{array}$ & $\begin{array}{l}\rightarrow \text { El sector portuario genera } \\
\text { USD } 1,8 \text { por cada dólar } \\
\text { destinado al gasto en el sector. }\end{array}$ \\
\hline $\begin{array}{l}\text { Braun, Xander \& } \\
\text { White }(2002)\end{array}$ & \begin{tabular}{|l} 
Este de Florida \\
Central, EEUU (1999)
\end{tabular} & $\begin{array}{l}\text { Puerto } \\
\text { Cañaveral }\end{array}$ & $\begin{array}{l}\rightarrow \text { Gasto de la Industria } \\
\text { de cruceros } \\
\rightarrow \text { Gasto de Pasajeros } \\
\rightarrow \text { Gastos de la tripulación }\end{array}$ & $\begin{array}{l}\rightarrow \text { USD 352,9 millones en el PBI. } \\
\rightarrow \text { USD } 225,4 \text { millones en } \\
\text { salarios. } \\
\rightarrow 14499 \text { empleos. }\end{array}$ \\
\hline Braun (1990) & $\begin{array}{l}\text { Este de Florida } \\
\text { Central, EEUU (1988) }\end{array}$ & $\begin{array}{l}\text { Puerto } \\
\text { Cañaveral }\end{array}$ & $\begin{array}{l}\rightarrow \text { Gastos de operación } \\
\text { del puerto }\end{array}$ & $\begin{array}{l}\rightarrow \text { USD } 709 \text { millones de VA en la } \\
\text { región. } \\
\rightarrow \text { USD } 225 \text { millones en salarios } \\
\text { en la región. } \\
\rightarrow 31265 \text { empleos en la región. }\end{array}$ \\
\hline $\begin{array}{l}\text { Mescon, Vozikis } \\
(1985)\end{array}$ & $\begin{array}{l}\text { Sureste Florida, EEUU } \\
(1982)\end{array}$ & Puerto de Miami & 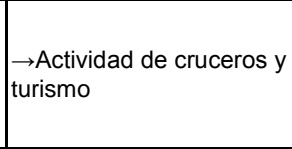 & $\begin{array}{l}\rightarrow \text { USD } 546 \text { millones en el PBI } \\
\text { de la región. } \\
\rightarrow \text { USD } 271 \text { millones en salarios } \\
\text { en la región. } \\
\rightarrow 21627 \text { empleos en la región. }\end{array}$ \\
\hline $\begin{array}{l}\text { De Rus, Tovar \& } \\
\text { Gonzáles (2009) }\end{array}$ & $\begin{array}{l}\text { Las Palmas, España } \\
(2005)\end{array}$ & $\begin{array}{l}\text { Puerto de las } \\
\text { Palmas }\end{array}$ & $\begin{array}{l}\rightarrow \text { Gastos en operación } \\
\rightarrow \text { Gastos de Industrias } \\
\text { conexas }\end{array}$ & $\begin{array}{l}\rightarrow 1,89 \% \text { VAB de la región. } \\
\rightarrow 1,19 \% \text { de la PEA en la región. }\end{array}$ \\
\hline $\begin{array}{l}\text { Martínez, } \\
\text { Malagón \& } \\
\text { Muñoz (2014) }\end{array}$ & $\begin{array}{l}\text { Cartagena, Colombia } \\
\text { (2011) }\end{array}$ & $\begin{array}{l}\text { Puerto de } \\
\text { Cartagena }\end{array}$ & $\begin{array}{l}\rightarrow \text { Gastos en operación } \\
\rightarrow \text { Gastos en Inversión en } \\
\text { Infraestructura }\end{array}$ & $\begin{array}{l}\rightarrow 3,1 \% \text { PBI de la ciudad de } \\
\text { Cartagena. } \\
\rightarrow \text { Se generó } 1866 \text { empleos } \\
\text { directos y un total de } 11366 \\
\text { empleos en toda la economía. }\end{array}$ \\
\hline
\end{tabular}

Nota: $V A=$ Valor Agregado; $P E A=P o b l a c i o ́ n$ Económicamente Activa.

Fuente: Elaboración propia.

\section{METODOLOGÍA}

Las estimaciones de impacto económico del sector portuario pueden analizarse desde numerosos métodos, suelen primar tres: los basados en las series de tiempo, en los Modelos de Equilibrio General Computable y en la MIP. La elección entre estas depende de los objetivos que se plantee el estudio, así como de la disponibilidad y fiabilidad de los datos. Chang, et al. (2016) y Wang \& Charles (2010) realizan un análisis de las ventajas y desventajas de estas metodologías. 
En el presente estudio se ha optado por estimar el impacto en base a la MIP. Si bien la metodología no contempla la dinámica de ajuste endógeno o modelice el comportamiento de los agentes, los análisis abarcan un profundo nivel de detalle, y permiten obtener una buena aproximación de los impactos en el valor agregado y empleo, así como las interrelaciones entre los diversos sectores económicos (Schuschny, 2005).

\subsection{La Matriz Insumo Producto peruana}

La MIP peruana utiliza cuatro matrices principales: la matriz de oferta total, la matriz de demanda intermedia, la matriz de valor agregado y la matriz de demanda final. (Ver Figura 2).

Figura 2

Estructura de los Cuadros de Oferta y Utilización

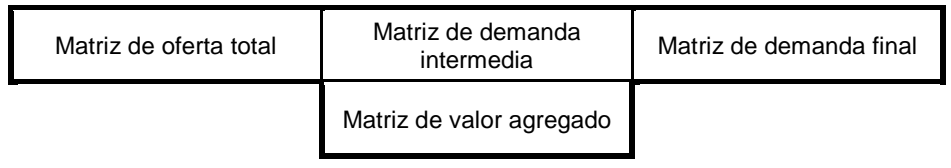

Fuente: Instituto Nacional de Estadística e Informática - INEI (2015). Elaboración Propia.

La matriz de oferta total comprende tanto la producción nacional como el componente importado; esta tendrá como destino el consumo intermedio (matriz de demanda intermedia) o el consumo final (matriz de demanda final). La matriz de valor agregado resume el pago que se realiza a los factores de producción.

Dado que se estima el impacto para el 2014, se usa la MIP de ese año, que está valorada a precios básicos, esto es, a diferencia de la valoración a precios del comprador, los márgenes del comercio y los gastos en transporte o fletes se registran como un sector aparte, pudiendo interactuar con los demás sectores de la economía. Asimismo, en la matriz de oferta se diferencia los impuestos indirectos y las subvenciones a los productos. Cabe mencionar que la valoración a precios básicos ofrece coeficientes técnicos más puros ya que están exentos de márgenes de distribución e impuestos indirectos (Schuschny, 2005).

Como se puede apreciar en la Figura 3, la matriz intermedia muestra el flujo de ventas del sector $i$ al sector $j\left(z_{i j}\right)$ y la matriz de demanda final muestra la utilización que se da a los bienes finales, pudiendo ser consumo privado y de las instituciones sin fines de lucro $\left(c_{i}\right)$, inversión $\left(i_{i}\right)$, consumo del gobierno $\left(g_{i}\right)$, variación de las existencias $\left(v e_{i}\right)$ o exportaciones $\left(e x_{i}\right)$. De esta forma, para el sector $i$, la oferta total es igual a la demanda total: 


$$
x_{i}+m_{i}=\sum_{j=1}^{n} z_{i j}+c_{i}+i_{i}+g_{i}+v e_{i}+e x_{i}
$$

Donde $x$ es el vector de producciones sectoriales $\mathrm{y} m$ el vector de importaciones por origen.

Figura 3

Modelo de Matriz Insumo-Producto Peruana

\begin{tabular}{|c|c|c|c|c|c|c|c|c|c|c|c|}
\hline & \multicolumn{4}{|c|}{ Demanda Intermedia } & \multicolumn{6}{|c|}{ Demanda Final } & \multirow[t]{2}{*}{\begin{tabular}{|c} 
Demanda \\
Total
\end{tabular}} \\
\hline & Sector 1 & Sector 2 & $\ldots$ & Sector $\mathbf{n}$ & & $c$ & $i$ & $g$ & $v e$ & ex $d$ & \\
\hline Sector 1 & $z_{11}$ & $z_{12}$ & $\ldots$ & $z_{1 n}$ & $\sum z_{1 i}$ & & $i_{1}$ & $g_{1}$ & $v e_{1}$ & $e x_{1} d_{1}$ & $X_{1}$ \\
\hline Sector 2 & $z_{21}$ & $z_{22}$ & $\ldots$ & $z_{2 n}$ & $\sum z_{2 i}$ & $c_{2}$ & $i_{2}$ & $g_{2}$ & $v e_{2}$ & $e x_{2} \quad d_{2}$ & $X_{2}$ \\
\hline$\cdots$ & $\cdots$ & $\cdots$ & $\cdots$ & $\cdots$ & $\cdots$ & $\cdots$ & $\ldots$ & $\cdots$ & $\cdots$ & $\cdots \quad \ldots$ & $\cdots$ \\
\hline Sector $n$ & $z_{n 1}$ & $z_{11}$ & $\ldots$ & $z_{n n}$ & $\sum z_{n i}$ & $c_{n}$ & $i_{n}$ & $g_{n}$ & $v e_{n}$ & $e x_{n} d_{n}$ & $X_{n}$ \\
\hline & $\sum z_{j 1}$ & $\sum z_{j 2}$ & $\ldots$ & $\sum z_{j n}$ & & & & & & & \\
\hline Valor agregado bruto & $v a_{1}$ & $v a_{2}$ & $\ldots$ & $v a_{n}$ & & & & & & & \\
\hline Salarios & $l_{1}$ & $l_{2}$ & $\ldots$ & $l_{n}$ & & & & & & & \\
\hline Beneficios & $r_{1}$ & $r_{2}$ & $\cdots$ & $r_{n}$ & & & & & & & \\
\hline $\begin{array}{l}\text { Impuestos y } \\
\text { Subvenciones }\end{array}$ & $t_{1}-s u b_{1}$ & $t_{2}-s u b_{2}$ & & $t_{n}-s u b_{n}$ & & & & & & & \\
\hline Producción bruta & $X_{1}$ & $X_{2}$ & $\ldots$ & & & & & & & & \\
\hline
\end{tabular}

Fuente: INEI (2015). Elaboración propia.

A partir de la demanda intermedia se puede obtener los coeficientes técnicos (o de requerimientos directos), $a_{i j}=\frac{z_{i j}}{X_{j}}$, que representan el consumo intermedio como porcentaje de la producción bruta que realiza el sector $j$ al sector $i$. Estos coeficientes conforman la matriz de requerimientos técnicos, $A_{n x n}$.

La utilización de la MIP supone que la tecnología para cada sector es la misma, y su producción es primaria ${ }^{4}$; asimismo asume rendimientos constantes a escala. En consecuencia, los coeficientes técnicos son constantes, por lo menos en el mediano plazo. Además, dado que la matriz trabaja con agregados monetarios, supone que los precios relativos también se mantienen constantes ${ }^{5}$.

\subsection{Modelo cerrado para una economía abierta}

Un modelo abierto considera los efectos del sector externo, como las exportaciones $(e x)$ e importaciones $(m)$. El primero tendrá su efecto en la

\footnotetext{
${ }^{4}$ La producción primaria es aquella que representa el giro del negocio; sin embargo, muchas empresas integran su producción verticalmente. Este inconveniente es subsanado en la matriz a medida que esta se construye con la máxima cantidad de sectores posibles.

${ }^{5}$ Este mismo supuesto se utiliza para el cálculo del PBI real, donde a partir de un año base, se supone que las variaciones de los precios en la economía se mantienen constantes.
} 
demanda final $(d)$, en tanto las importaciones constituyen la parte de la oferta que es satisfecha con productos importados, pudiendo ser utilizados tanto para los consumos intermedios como para el consumo final. La oferta total y la demanda total satisfacen la siguiente identidad:

$$
x+m=A x+d
$$

Se asume que las importaciones son una parte fija de la producción, $m=$ $M x$, tal que se define la matriz diagonal, $M$, cuyos elementos están conformados por los coeficientes de importación, $\mathrm{cm}=\frac{m}{x}$.

$$
M=\left[\begin{array}{ccc}
c m_{1} & \cdots & 0 \\
\vdots & \ddots & \vdots \\
0 & \cdots & c m_{i}
\end{array}\right]
$$

De esta forma, la solución a la ecuación (2) está dada por:

$$
x=(I+M-A)^{-1} * d
$$

La matriz $(I+M-A)^{-1}$ es conocida como Matriz de Leontief. Esta matriz captura los impactos directos e indirectos. Los impactos directos agrupan la producción sectorial necesaria para satisfacer una variación de la demanda final (Martí \& Puertas, 2012); este impacto puede estimarse directamente a través de las cuentas registrales de los agentes que operan el puerto. La demanda intermedia emplazada por el primer impacto requerirá, a su vez, consumos intermedios de otros sectores en rondas sucesivas; el aglutinamiento de estos impactos suele denominarse impactos indirectos (De Rus, Tovar \& Gonzáles, 2009).

Un modelo cerrado para una economía abierta incluirá la demanda generada por las rentas que han obtenido los agentes económicos en las diferentes etapas del proceso productivo, ya que estos agentes también consumen y estimulan la demanda. Una forma sencilla de endogenizar el consumo es suponer que el consumo está en función al valor agregado $(v a)^{6}$, que a su vez es una proporción del producto nacional, $v a=v^{\prime} x^{\prime}$ (INEI, 2001). Seguidamente, a través de la proporción marginal a consumir keynesiana ${ }^{7}, b$, definimos al consumo como $C=b * t_{c} * v^{\prime} * x$, donde $t_{c}$ es el vector de proporciones sectoriales del consumo. Por extensión, la ecuación (4) es:

$$
x=\left(I+M-A-b * t_{c} * v^{\prime}\right)^{-1} * d
$$

\footnotetext{
${ }^{6}$ El valor agregado bruto está conformado por los sueldos y salarios, las contribuciones sociales a los empleadores, el excedente bruto de explotación, los ingresos mixtos brutos y los subsidios netos de otros impuestos a la producción.

${ }^{7}$ Para la estimación de la propensión marginal a consumir Keynesiana se emplea un análisis de cointegración y un modelo de corrección de errores, con datos trimestrales desde el primer trimestre de 2003 hasta el tercer trimestre de 2017 a fin de obtener la relación de largo plazo entre las variables ingreso disponible y consumo privado, puesto que no existen mediciones confiables de dicha propensión para el caso peruano.
} 
De esta forma, $R=\left(I+M-A-b * t_{c} * v^{\prime}\right)^{-1}$ es la matriz de Leontief para un modelo cerrado para una economía abierta. En este modelo, un cambio en la demanda final genera una variación en la producción nacional equivalente a:

$$
\Delta x=R * \Delta d
$$

A partir del modelo cerrado, es posible obtener los denominados Multiplicadores Keynesianos de la Producción (MKP), estos resultan de la suma de elementos de cada columna de la matriz $R$ de cada sector, tal como:

$$
M K P=\left[R_{1}+R_{2}+R_{3}+\cdots+R_{n}\right]
$$

En tanto los Multiplicadores Keynesianos del Ingreso (MKI) para el sector $i$ :

$$
M K I_{i}=v_{i}^{\prime} * R_{i}
$$

Y los Multiplicadores Keynesianos del Empleo (MKE) para el sector $i$ :

$$
M K I_{i}=\operatorname{cem}_{i}^{\prime} * R_{i}
$$

Donde $c m e^{\prime}$ es el vector que contiene los coeficientes de empleo.

El análisis de los impactos puede resumirse en la Figura 4. Como se aprecia, el impacto total es la suma de los impactos directos, indirectos e inducidos según lo explicado líneas arriba. Es importante mencionar que el impacto que se estima en el presente estudio considera los producidos por la actividad de los concesionarios en el puerto, que incluye las inversiones que se ejecutan en los mismos, mas no lo producido por las actividades de las industrias relacionadas a la actividad portuaria, como el de las líneas navieras, agencias marítimas, etc.

Figura 4

Análisis del Impacto Portuario

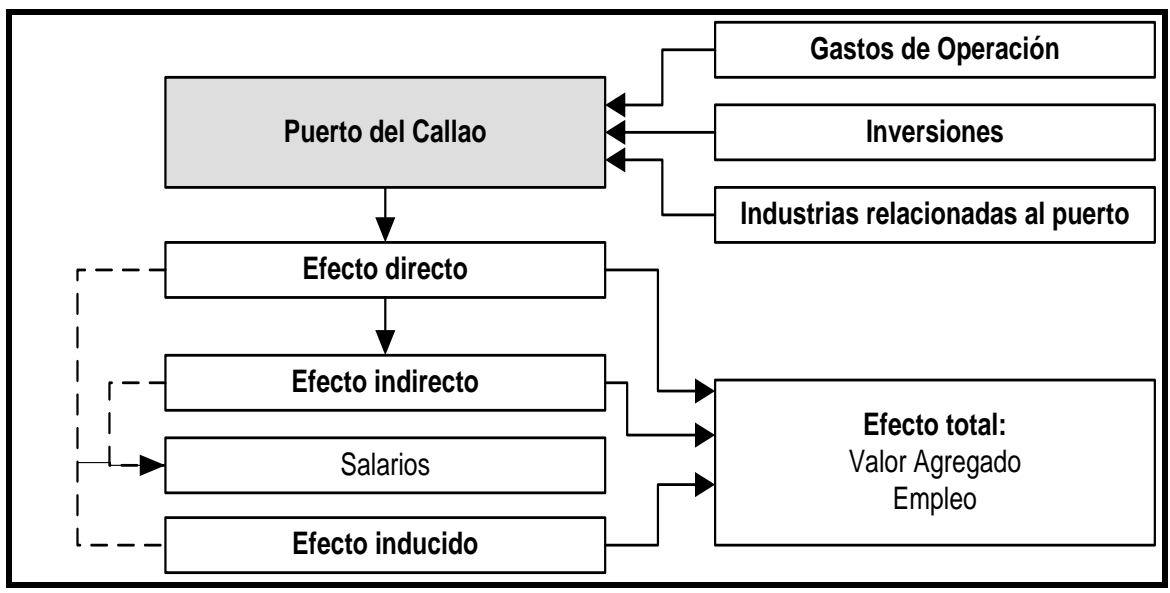

Fuente: Elaboración propia. 


\section{DATOS}

La medición de Impacto económico a través de la MIP considera exógena las variaciones de los componentes de la demanda final, como los gastos destinados a la operación e inversiones ejecutadas por la TMN, la TMS, y la TCM. Los datos correspondientes a las inversiones se obtuvieron de los informes de revisión de las valorizaciones, realizadas por el Ositran, mientras que los datos por operación se obtuvieron de los estados financieros auditados de cada empresa prestadora para el año 2014. Estos datos se llevaron a valores constantes de 2007 a fin de guardar coherencia con la MIP peruana de 2014.

Como se aprecia en la Tabla 4, en cuanto a las inversiones ejecutadas en 2014, la concesionaria de la TMS ejecutó una inversión de USD 7,2 millones en adquisición de equipos como inversión complementaria a la primera fase ${ }^{8}$. En ese mismo año, la TMN invirtió alrededor de USD 185,6 millones a la ejecución de obras civiles, que comprendían obras de dragado y relleno, así como la modernización de los muelles 5 y 11; adicionalmente se adquirieron equipos diversos como grúas pórtico de muelle panamax y post-panamax, tractores, remolques, y equipos menores valorizados en USD 154,7 millones. Por último, el concesionario de la TCM ejecutó el último tramo de las inversiones obligatorias establecidas en el Contrato de Concesión, para lo cual invirtió USD 3,5 millones en equipos y USD 2,9 millones en construcción de nueva infraestructura. De este modo, con el inicio de operaciones de la TCM, el puerto del Callao en su conjunto operaba en toda su capacidad.

\section{Tabla 4}

Inversiones y Gastos en Operación de las Terminales del Puerto del Callao, 2014 (Miles de dólares, a precios constantes de 2007)

\begin{tabular}{|l|c|c|c|}
\hline \multicolumn{1}{|c|}{ Terminal } & Inversión & Operación & Total \\
\hline Terminal Muelle Norte & 328745 & 60390 & 389135 \\
\hline Terminal Muelle Sur & 5881 & 55316 & 61198 \\
\hline Terminal de Concentrados de Minerales & 10423 & 11117 & 21541 \\
\hline Total & $\mathbf{3 4 5 0 4 9}$ & $\mathbf{1 2 6 8 2 4}$ & $\mathbf{4 7 1 8 7 3}$ \\
\hline
\end{tabular}

Fuente: Informes de Valorización de Inversiones y Estados Financieros Auditados de las Concesionarias. Elaboración propia.

De acuerdo al INEI (2017), el proceso de clasificación sectorial de las inversiones y los gastos en operación realizados por las concesionarias debe enumerar primero las actividades que involucran las inversiones e identificar el

\footnotetext{
${ }^{8}$ Cabe señalar que, como parte del segundo factor de competencia en el proceso de licitación del muelle, el concesionario estuvo obligado a ceder a la Autoridad Portuaria Nacional (APN) USD 144 millones para la ejecución de obras en áreas comunes en el puerto como Inversiones Complementarias Adicionales (ICA), de las cuales USD 31 millones fueron entregadas al fideicomiso en 2014.
} 
componente que representa el mayor valor agregado. Posteriormente, en base a este componente escoger el sector según el CIIU Revisión 4, así como la clasificación económica en las cuentas nacionales.

\section{RESULTADOS}

La Figura 5 muestra los multiplicadores keynesianos sectoriales del modelo cerrado para una economía abierta. Cabe precisar que los multiplicadores del producto brindan información respecto a la capacidad de un sector para generar producción bruta total, que comprende tanto el consumo intermedio como el valor agregado. Los multiplicadores del ingreso, en cambio, tienen en cuenta solo el valor agregado. Así, se evidencia que existe una alta correlación inversa entre la capacidad de un sector en generar valor agregado respecto a su coeficiente de importación. Esto podría explicarse por el hecho que los sectores con un alto componente importado emplean menos mano de obra nacional y no estimulan la producción interna, limitando su potencial para generar valor agregado.

Figura 5

Multiplicadores Keynesianos, 2014

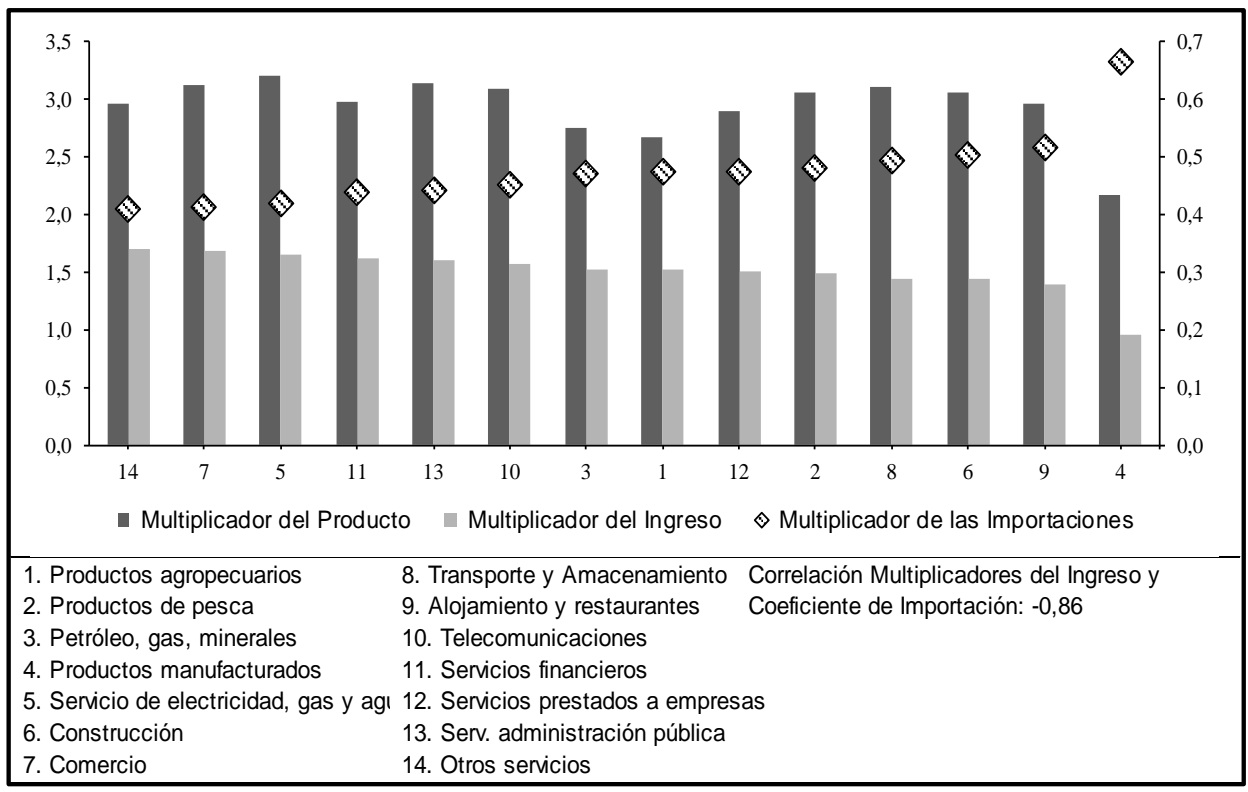

Fuente: Elaboración propia.

Por su parte, el Multiplicador del Ingreso resulta ser el más importante debido a su estrecha relación con el PBI. Los sectores con mayor capacidad de generar valor agregado son el sector comercio, el sector energético y el de servicios financieros. El primero, que es intensivo en mano de obra, lo hace a través de 
sueldos y salarios, en tanto los dos restantes a través del excedente de explotación. Cabe resaltar que el sector agrícola es el que genera más empleos (437 empleos por cada millón de dólares invertidos), aunque su capacidad de generar valor agregado es limitada. En el caso del sector transportes, por ejemplo, una inversión adicional de USD 1,0 millón en dicho sector incrementaría en forma agregada -entre efectos directos, indirectos e inducidos- la producción bruta en USD 3,1 millones, las importaciones en USD 0,49 millones, los ingresos en USD 1,45 millones y generaría 159 empleos.

La TMN obtuvo un mayor impacto de la inversión y operación en el valor agregado y la generación de empleo (Ver Tabla 5). Este resultado se debe a que la inversión ejecutada en dicha terminal representó el 95\% de la inversión ejecutada en todo el puerto y que dicha terminal realizó un mayor gasto en operación para dicho año en comparación con las otras terminales. Así, la TMN invirtió USD 328,8 millones, el cual produjo un impacto en el valor agregado de USD 389,5 millones que representa el $0,26 \%$ del total del valor agregado. Asimismo, el impacto en la generación de empleo asciende en 44,5 mil empleos y representa el 0,27\% de la Población Económicamente Activa ${ }^{9}$ (PEA). Por su parte, el gasto de USD 60,4 millones en la operación de la TMN produce un impacto de USD 89,5 millones en el valor agregado $(0,06 \%)$ y la generación de 9,6 mil empleos, $0,06 \%$ de la PEA.

Tabla 5

Impactos de la Inversión y Operación de la Terminal Muelle Norte, 2014

\begin{tabular}{|c|c|c|c|c|}
\hline \multirow{2}{*}{} & \multicolumn{2}{|c|}{ Inversiones } & \multicolumn{2}{c|}{ Gasto en Operación } \\
\cline { 2 - 5 } & $\begin{array}{c}\text { Valor Agregado } \\
\text { (miles de USD) }\end{array}$ & $\begin{array}{c}\text { Empleos } \\
\text { (en miles) }\end{array}$ & $\begin{array}{c}\text { Valor Agregado } \\
\text { (miles de USD) }\end{array}$ & $\begin{array}{c}\text { Empleos } \\
\text { (en miles) }\end{array}$ \\
\hline Input & $\mathbf{3 2 8 7 4 5}$ & - & $\mathbf{6 0 3 9 0}$ & - \\
\hline Output & & & & 3,5 \\
\hline Efecto Directo & 187325 & 19,1 & 41069 & 0,9 \\
\hline Efecto Indirecto & 22254 & 2,8 & 7245 & 5,2 \\
\hline Efecto Inducido & 179947 & 22,5 & 41175 & $\mathbf{9 , 6}$ \\
\hline Efecto Total & $\mathbf{3 8 9 5 2 6}$ & $\mathbf{4 4 , 5}$ & $\mathbf{8 9 4 8 9}$ & $\mathbf{0 , 0 6 \%}$ \\
\hline Porcentaje del total ${ }^{\mathbf{1 /}}$ & $\mathbf{0 , 2 6 \%}$ & $\mathbf{0 , 2 7 \%}$ & $\mathbf{0 , 0 6 \%}$ & \\
\hline
\end{tabular}

1/ Porcentaje del total de Valor Agregado Bruto y Población Económicamente Activa del 2014.

Fuente: Elaboración propia.

Por su parte, al 2014 la TMS al haber ejecutado sus inversiones obligatorias especificadas en el Contrato de Concesión antes del 2014, las inversiones ejecutadas en dicho año fueron menores a las realizadas por la TMN (USD 5,9 millones). Los impactos de tales inversiones en el valor agregado fueron de

${ }^{9}$ Cabe precisar que los empleos generados comprenden tanto los asalariados como los no asalariados y en el 2014, el 49\% de la PEA ocupada era no asalariada (INEI, 2015). 
USD 5,7 millones $(0,004 \%)$ y el impacto en la generación de empleo de 700 personas empleadas $(0,004 \%$ de la PEA). Por su parte, los impactos generados por los USD 55,3 millones en gastos de operación de la TMS fueron de USD 82,6 millones en valor agregado que representan el 0,06\%, y de 8,2 mil personas empleadas, es decir el 0,05\% de la PEA.

\section{Tabla 6}

Impactos en de la Inversión y Operación de la Terminal Muelle Sur, 2014

\begin{tabular}{|c|c|c|c|c|}
\hline & \multicolumn{2}{|c|}{ Inversiones } & \multicolumn{2}{|c|}{ Gasto en Operación } \\
\hline & $\begin{array}{l}\text { Valor Agregado } \\
\text { (miles de USD) }\end{array}$ & $\begin{array}{l}\text { Empleos } \\
\text { (en miles) }\end{array}$ & $\begin{array}{l}\text { Valor Agregado } \\
\text { (miles de USD) }\end{array}$ & $\begin{array}{l}\text { Empleos } \\
\text { (en miles) }\end{array}$ \\
\hline Input & 5881 & - & 55316 & - \\
\hline \multicolumn{5}{|l|}{ Output } \\
\hline Efecto Directo & 2869 & 0,4 & 38780 & 2,8 \\
\hline Efecto Indirecto & 176 & 0,0 & 5790 & 0,7 \\
\hline Efecto Inducido & 2612 & 0,3 & 38039 & 4,8 \\
\hline Efecto Total & 5658 & 0,7 & 82609 & 8,2 \\
\hline Porcentaje del total $^{1 /}$ & $0,004 \%$ & $0,004 \%$ & $0,06 \%$ & $0,05 \%$ \\
\hline
\end{tabular}

1/ Porcentaje del total de Valor Agregado Bruto y Población Económicamente Activa del 2014.

Fuente: Elaboración propia.

En lo que respecta al impacto de las inversiones ejecutadas por la TCM, la inversión de USD 10,4 millones generaron USD 13,8 millones en valor agregado equivalentes a $0,01 \%$ del valor agregado, y $1,5 \mathrm{mil}$ personas empleadas que representa el $0,01 \%$ de la PEA. Por otro lado, el impacto de USD 11,1 millones en gastos de operación generaron USD 17,3 millones en valor agregado $(0,01 \%)$ y 1,6 mil personas empleadas, que representan el $0,01 \%$ de la PEA.

Tabla 7

Impactos en de la Inversión y Operación de la Terminal de Concentrados de Minerales, 2014

\begin{tabular}{|l|c|c|c|c|}
\hline \multirow{2}{*}{} & \multicolumn{2}{|c|}{ Inversiones } & \multicolumn{2}{c|}{ Gasto en Operación } \\
\cline { 2 - 5 } & $\begin{array}{c}\text { Valor Agregado } \\
\text { (miles de USD) }\end{array}$ & $\begin{array}{c}\text { Empleos } \\
\text { (en miles) }\end{array}$ & $\begin{array}{c}\text { Valor Agregado } \\
\text { (miles de USD) }\end{array}$ & $\begin{array}{c}\text { Empleos } \\
\text { (en miles) }\end{array}$ \\
\hline Input & $\mathbf{1 0 4 2 3}$ & - & $\mathbf{1 1} \mathbf{1 1 7}$ & - \\
\hline Output & & & & 0,5 \\
\hline Efecto Directo & 6526 & 0,6 & 8285 & 0,1 \\
\hline Efecto Indirecto & 884 & 0,1 & 1011 & 1,0 \\
\hline Efecto Inducido $^{\text {Efecto Total }}$ & 6357 & 0,8 & 7957 & $\mathbf{1 , 6}$ \\
\hline Porcentaje del total $^{\mathbf{1 1}}$ & $\mathbf{1 3 7 6 7}$ & $\mathbf{1 , 5}$ & $\mathbf{1 7 2 5 3}$ & $\mathbf{0 , 0 1 \%}$ \\
\hline
\end{tabular}

1/ Porcentaje del total de Valor Agregado Bruto y Población Económicamente Activa del 2014.

Fuente: Elaboración propia. 
En promedio, los impactos directos, conformado por la generación de ingresos resultado de la producción de todos los sectores para satisfacer la demanda final, representaron el $48 \%$ del impacto total. Los efectos indirectos, correspondientes a la producción necesaria para satisfacer los requerimientos de demanda intermedia sucesivas, no tienen mucho potencial para generar valor agregado y representaron solo el 6\%. Los efectos inducidos, generados por el multiplicador keynesiano, tienen un gran potencial para generar valor agregado, este contribuyó con el 46\% del efecto total. Estos resultados son acordes con la teoría, puesto que los Modelos basados en la MIP suponen que la transición al equilibrio final sucede rápidamente (Mariña, 1993), esto es, que los efectos indirectos, que comprende los impactos generados por los requerimientos de inputs intermedios en rondas sucesivas distintas a la primera ronda, son exponencialmente decrecientes.

Respecto a los efectos marginales por terminal, la Tabla 8 muestra el impacto de un dólar destinado al gasto en operación o en inversión en el valor agregado de las terminales portuarias del Callao. Así, independientemente de los montos, una inversión de USD 1,0 genera USD 1,18 en la TMN, USD 0,96 en la TMS y USD 1,32 en la TCM en términos de valor agregado. Estas diferencias podrían explicarse por la heterogeneidad de la inversión en cada terminal, así como la naturaleza de la concesión. En ese sentido, considerando que el TMN es un proyecto brownfield, más del 50\% de la inversión ejecutada en el 2014 correspondió a la adquisición de productos manufacturados, que para el sector portuario suelen contener un alto componente importado, limitando así su capacidad para generar valor agregado. En tanto, la inversión en las terminales TCM y TMS, siendo ambas greenfield, involucraron más al sector construcción, que es intensivo en mano de obra.

Tabla 8

Impacto de un dólar destinado al gasto en operación o en inversión, 2014 (Dólares, a precios constantes de 2007)

\begin{tabular}{|l|c|c|}
\hline \multicolumn{1}{|c|}{ Terminal } & Inversión Ejecutada & Gastos en Operación \\
\hline Terminal Muelle Norte & 1,18 & 1,48 \\
\hline Terminal Muelle Sur & 0,96 & 1,49 \\
\hline Terminal de Concentrados de Minerales & 1,32 & 1,55 \\
\hline Promedio & $\mathbf{1 , 1 6}$ & $\mathbf{1 , 5 1}$ \\
\hline
\end{tabular}

Fuente: Elaboración propia.

Entre tanto, destinar USD 1,0 en la operación de los puertos produce resultados similares, entre USD 1,48 y USD 1,55. En general, un USD 1,0 en la operación de un puerto contribuye más a la generación de valor agregado que la inversión en los mismos. Este resultado podría explicarse por el hecho que la operación del puerto requiere de sectores intensivos en mano de obra, en tanto la 
inversión suele necesitar de sectores como la construcción y manufactura, siendo este último un sector que contiene un alto componente importado.

Finalmente, la Tabla 9 muestra los impactos de la inversión y los gastos de operación en los 14 sectores de la economía peruana: productos agropecuarios; productos de pesca; petróleo, gas, minerales; productos manufacturados; servicio de electricidad, gas y agua; construcción; comercio; transporte y almacenamiento; alojamiento y restaurantes; telecomunicaciones; servicios financieros; servicios prestados a empresas; servicios de administración pública; y otros servicios. Cabe señalar que, si bien en un modelo cerrado el efecto inducido propaga en mayor medida los efectos hacia todos los sectores, el resultado final depende del sector donde se dé la variación en la demanda final.

\section{Tabla 9}

Impacto total de la Inversión y Operación en las Terminales del Puerto del Callao, por sectores económicos, 2014

\begin{tabular}{|l|c|c|c|c|c|c|}
\hline \multirow{2}{*}{ Sector Económico } & \multicolumn{2}{|c|}{ Inversiones (a) } & \multicolumn{2}{c|}{ Gasto en Operación (b) } & \multicolumn{2}{c|}{ Impacto Total (c)=(a)+(b) } \\
\cline { 2 - 7 } & $\begin{array}{l}\text { Valor Agregado } \\
\text { (miles de USD) }\end{array}$ & $\begin{array}{c}\text { Empleo } \\
\text { (miles) }\end{array}$ & $\begin{array}{c}\text { Valor Agregado } \\
\text { (miles de USD) }\end{array}$ & $\begin{array}{c}\text { Empleo } \\
\text { (miles) }\end{array}$ & $\begin{array}{c}\text { Valor Agregado } \\
\text { (miles de USD) }\end{array}$ & $\begin{array}{c}\text { Empleo } \\
\text { (miles) }\end{array}$ \\
\hline 1. Productos agropecuarios & 39712 & 18,7 & 12 221 & 5,8 & 51933 & 24,5 \\
\hline 2. Productos de pesca & 2673 & 0,4 & 865 & 0,1 & 3538 & 0,6 \\
\hline 3. Petróleo, gas, minerales & 34128 & 0,4 & 8140 & 0,1 & 42269 & 0,5 \\
\hline 4. Productos manufacturados & 114003 & 8,4 & 28474 & 2,1 & 142477 & 10,5 \\
\hline $\begin{array}{l}\text { 5. Servicio de electricidad, gas y } \\
\text { agua }\end{array}$ & 9291 & 0,2 & 5926 & 0,1 & 15216 & 0,3 \\
\hline 6. Construcción & 66331 & 5,9 & 505 & 0,0 & 66836 & 6,0 \\
\hline 7. Comercio & 3972 & 0,6 & 4803 & 0,8 & 8774 & 1,4 \\
\hline 8. Transporte y Almacenamiento & 18753 & 1,7 & 38411 & 3,4 & 57164 & 5,1 \\
\hline 9. Alojamiento y restaurantes & 12614 & 2,8 & 6265 & 1,4 & 18879 & 4,3 \\
\hline 10. Telecomunicaciones & 16956 & 0,5 & 10252 & 0,3 & 27208 & 0,9 \\
\hline 11. Servicios financieros & 17012 & 0,3 & 17306 & 0,3 & 34317 & 0,7 \\
\hline 12. Servicios prestados a empresas & 31892 & 2,5 & 28156 & 2,2 & 60049 & 4,7 \\
\hline $\begin{array}{l}\text { 13. Servicios de administración } \\
\text { pública }\end{array}$ & 461 & 0,0 & 7420 & 0,6 & 7881 & 0,6 \\
\hline 14. Otros servicios & 41153 & 4,1 & 20608 & 2,0 & 61760 & 6,1 \\
\hline Impacto total & $\mathbf{4 0 8 9 5 1}$ & $\mathbf{4 6 , 6}$ & $\mathbf{1 8 9} \mathbf{3 5 0}$ & $\mathbf{1 9 , 4}$ & $\mathbf{5 9 8} \mathbf{3 0 1}$ & $\mathbf{6 6 , 0}$ \\
\hline Porcentaje del Total ${ }^{1 /}$ & $\mathbf{0 , 2 7 \%}$ & $\mathbf{0 , 2 8 \%}$ & $\mathbf{0 , 1 3 \%}$ & $\mathbf{0 , 1 2 \%}$ & $\mathbf{0 , 4 0 \%}$ & $\mathbf{0 , 4 0 \%}$ \\
\hline
\end{tabular}

1/ Porcentaje del total de Valor Agregado Bruto y Población Económicamente Activa del 2014.

Fuente: Elaboración propia.

Así, las inversiones ejecutadas en las terminales portuarias del Callao tuvieron un impacto ascendente a USD 144,0 millones en el sector manufactura y USD 66,3 millones en el sector construcción, con relación al valor agregado; generando 18,7 mil empleos en el sector de agricultura, 8,4 mil empleos en el sector manufactura y 5,9 mil empleos en el sector construcción. Los gastos destinados a la operación de las terminales portuarias, tuvieron un impacto en el 
valor agregado en el sector transporte y almacenamiento de USD 38,4 millones, USD 28,5 millones en el sector manufactura y USD 28,2 millones en el sector de servicios prestados a empresas, lo que a su vez generó 5,8 mil empleos en el sector agricultura y 3,4 mil empleos en el sector transporte y almacenamiento.

\section{CONCLUSIONES}

El objetivo del estudio es medir el impacto en el valor agregado y la generación de empleo de los gastos destinados a la operación e inversiones ejecutadas en las terminales portuarias del Callao para el año 2014. Para tal fin, se utiliza un modelo cerrado para una economía abierta, basada en la Matriz Insumo - Producto peruana de 2014 que cuenta con 14 sectores económicos.

Desde el punto de vista empírico, el presente estudio destaca por ser la primera vez que se analiza el impacto de los gastos destinados a la operación de un puerto y la inversión ejecutada en el mismo. Asimismo, es el primer estudio en el Perú que mide el impacto económico de la operación e inversión portuaria, mediante el uso de la Matriz Insumo-Producto.

La evidencia empírica, muestra que las inversiones y el gasto en operación de las terminales portuarias del Callao para el 2014 generaron un impacto total de USD 598 millones y 66 mil empleos, siendo la TMN la que presentó un mayor impacto total. En particular, la inversión de USD 328,8 millones en la TMN; que comprendieron la ejecución de obras civiles como las dragado y relleno, la modernización de los muelles 5 y 11, y la adquisición de equipos diversos como grúas pórtico de muelle panamax y post-panamax, tractores, remolques; generaron un impacto de USD 389,5 millones en el valor agregado y la creación de 44,5 mil empleos. Por su parte, los gastos en operación de la TMN, que ascendieron a USD 60,4 millones, generaron USD 89,5 millones en valor agregado y 9,6 mil empleos. En el caso de la TMS, la inversión de USD 5,9 millones en adquisición de equipos diversos, conllevaron a un impacto de USD 5,7 millones en el valor agregado y 700 empleos; mientras que los USD 55,3 millones de gastos de operación generaron un impacto de 82,7 millones en el valor agregado y 8,2 mil empleos. Por último, la inversión de USD 10,4 millones en equipos y en la construcción de nueva infraestructura por parte de la TCM, generó un impacto de USD 17,3 millones en el valor agregado y 1,6 mil empleos; mientras que el gasto en operación de USD 11,1 millones derivaron en USD 17,3 millones en valor agregado y 1,6 mil empleos.

Respecto a los impactos marginales, la TCM obtuvo un mayor impacto. Así, una inversión de USD 1,0 genera en la TCM conlleva a USD 1,32 en valor agregado, mientras que USD 1,0 en gasto de operación genera USD 1,55. En lo que respecta a las otras terminales, el impacto marginal de la inversión asciende en USD 1,18 en la TMN, y USD 0,96 en la TMS, mientras que el de gasto de operación genera un impacto marginal de USD 1,48 en la TMN y USD 1,49 en 
la TMS. El mayor impacto de los gastos de operación en comparación de la inversión podría explicarse por el hecho que la operación del puerto requiere de sectores intensivos en mano de obra, en tanto la inversión en puertos suele caracterizarse por necesitar de sectores como la construcción y manufactura, siendo este último un sector que contiene un alto componente importado, especialmente en la industria portuaria.

Respecto a los impactos sectoriales, las inversiones en las terminales portuarias del Callao estimularon más los sectores de manufactura y construcción con relación al valor agregado, y los sectores de agricultura, manufactura y construcción con relación al empleo. Respecto a la generación de valor agregado producto del gasto en la operación del puerto, los sectores de transporte y almacenamiento, manufactura y servicios prestados a empresas fueron los más favorecidos, en tanto los sectores de agricultura, y transporte y almacenamiento demandaron más empleo.

Por lo tanto, se sugiere a los entes competentes considerar estos resultados para realizar una mejor planificación en el desarrollo del sector, de manera que se continúe con los procesos de concesión, como el de la terminal portuaria de Salaverry en Trujillo, la terminal portuaria de Ilo en Moquegua, la nueva terminal portuaria de San Juan de Marcona en Nasca y la nueva terminal portuaria de contenedores de Chimbote en Áncash. Dichos procesos de concesión permitirán que se aceleren las inversiones y se intensifique la operación de las terminales lo que retribuirá en mayor valor agregado y empleo en las zonas de influencia.

Finalmente, futuras investigaciones deberían de ser orientadas a realizar una Matriz Insumo - Producto mediante cuentas satélites para el sector portuario, con la finalidad de analizar con mayor detalle la interrelación de dicho sector con los otros sectores de la economía.

\section{REFERENCIAS BIBLIOGRÁFICAS}

ACCIARO, M. (2008). "The role of Ports in the Development of Mediterranean Islands: The Case of Sardinia". Revista Internazionale di Economia dei Trasporti, 35(3), pp. 295323.

ARTAL, A.; GÓMEZ, J.M.; NAVARRO, J.M. \& RAMOS, J.M. (2016). "Estimating the economic impact of a port through regional input-output tables: Case study of the Port of Cartagena (Spain)". Maritime Economics \& Logistics, 18, pp. 371-390.

AUTORIDAD PORTUARIA NACIONAL (2010). Plan Maestro del Terminal Portuario del Callao. APN: Lima.

BRAUN, B.M. (1990). "Measuring the influence of public authorities through economic impact analysis: the case of port Canaveral". Policy Studies Journal, 18(4), pp. 10331044. 
BRAUN, B.M.; XANDER, J.A. \& WHITE, K.R. (2002). "The impact of the cruise industry on a region's economy: a case study of Port Canaveral, Florida". Tourism Economic, 8(3), pp. 281-288.

CEPAL (2018). Ranking de puertos. Los Top 20 en América Latina y el Caribe en 2016. http://perfil.cepal.org///es/portmovements_classic.html [Último acceso: abril de 2018].

CHANG, V. \& TOVAR, B. (2014a). "Efficiency and productivity changes for Peruvian and Chilean ports terminals: a parametric distance functions approach". Transport Policy, 31, pp. 83-94.

CHANG, V. \& TOVAR, B. (2014b). "Drivers explaining the inefficiency of Peruvian and Chilean ports terminals". Transportation Research Part E, 67, pp. 190-203.

CHANG, V. \& TOVAR, B. (2017a). "Heterogeneity unobserved and efficiency: A latent class model for west coast of south pacific port terminals". Transportation Research Part A, 103, pp. 118-134.

CHANG, V. \& TOVAR, B. (2017b). "Metafrontier analysis on productivity for West Coast of South Pacific terminals". Transportation Research Part A, 103, pp. 118-134.

CHANG, Y.T.; PARK, H.; LIU, S.M. \& ROH, Y. (2016). "Economic impact of cruise industry using regional input-output analysis: a case study of Incheon". Maritime Economics \& Logistics, 43(1), pp. 1-18.

CHANG, Y.T.; SHIN, S.H. \& LEE.P.T.W. (2014). "Economic impact of port sectors on South African economy: An input analysis". Transport Policy, 35, pp. 333-340

DANIELIS, R. \& GREGORI, T. (2013). "An input-output-based methodology to estimate the economic role of a port: The case of the port system of the Friuli Venezia Giulia Region, Italy". Maritime Economics \& Logistics, 15, pp.222-255.

De RUS, G.; TOVAR, B. \& GONZALES, M. M. (2009). Impacto Económico del Puerto de las Palmas. Navarra: Editorial Aranzadi.

ESPINOZA, R.; HERNÁNDEZ-AVILA, M.; NARCISO, J.; CASTAGAÑA, C.; MOSCOSO, S.; ORTIZ, G. CARBAJAL, L.; WEGNER, S. \& NOONAN, G. (2003). "Determinants of blood-lead levels in children in Callao and Lima metropolitan area". Salud Pública de México, 45, pp. 209-219.

INSTITUTO NACIONAL DE ESTADÍSTICA E INFORMÁTICA (2001). Multiplicadores de la Economía Peruana, INEI: Lima.

INSTITUTO NACIONAL DE ESTADÍSTICA E INFORMÁTICA (2015). Perú: Cuentas Nacionales 1950-2014. Lima: INEI.

INSTITUTO NACIONAL DE ESTADÍSTICA E INFORMÁTICA (2017). Perú: Cuentas Nacionales 1950-2016. Lima: INEI.

KWAK, S-J; YOO, S-H. \& CHANG, J-I. (2005). "The role of maritime industry in the Korean national economy: an input-output analysis". Marine Policy, 29, pp. 371-383.

LEE, T-C; LEE, P.T-W. \& CHEN, T. (2012). "Economic Impact Analysis of Port Development on the South African Economy". South African of Economics, 80(2), pp. 228-245.

MARIÑA, A. (1993). Insumo-Producto: Aplicaciones básicas al análisis económico estructural. México D.F.: Universidad Autónoma Metropolitana.

MARTíNEZ, A.; MALAGÓN, J. \& MUÑOZ, I. (2014). Impacto económico y social del puerto de Cartagena. Bogotá: FEDESARROLLO.

MESCON, T. \& VOZIKIS, G. (1985). "The Economic Impact of Tourism at The Port of Miami". Annals of Turism Research, 12, pp. 515-528. 
MUSSO, E.; BENACCHIO \& M. Y FERRARI, C. (2000). "Ports and employment in port cities". International Journal of Maritime Economics, 2(4), pp. 283-311.

ORGANISMO SUPERVISOR DE LA INVERSIÓN EN INFRAESTRUCTURA DE TRANSPORTE DE USO PÚBLICO - OSITRAN (2018). "Boletín Estadístico - Febrero 2018". Lima: Ositran.

PARK, J.S. \& SEO, Y.J. (2016). "Impact of seaports on the regional economies in South Korea: Panel evidence from the augmented Solow model". Transportation Research E, 85, pp. 107-119.

SCHUSCHNY, A.R. (2005). Tópicos sobre el Modelo de Insumo-Producto: teoría y aplicaciones. Chile: Cepal.

MARTÍ, M. L., PUERTAS, R. (2012). "Impacto económico de la celebración de un evento deportivo: Campeonato del Mundo de MotoGP en Valencia". Estudios de Economía Aplicada, 30(2), pp. 683-702.

SHAN, J., YU, M. \& LEE, C.Y. (2014). "An empirical investigation of the seaport's economic impact: Evidence from major ports in China". Transportation Research Part E, 69, pp. 41-53.

SONG, L. \& GEENHUIZEN, M. (2014). "Port Infrastructure investment and regional economic growth in China: Panel evidence in port regions and provinces". Transport Policy, 36, pp. 173-183.

UNCTAD (2015). "Informe Sobre el Transporte Marítimo 2015". Conferencia de las Naciones Unidas sobre el comercio y desarrollo. Disponible en: http://unctad.org/es/PublicationsLibrary/rmt2015_es.pdf [Último acceso: 1/04/2018].

WANG, J. \& CHARLES, M. (2010). "IO Based Impact Analysis: A Method for Estimating the Economic Impacts by Different Transport Infrastructure Investments is Australia". Australasian Transport Research Forum, Canberra. Disponible en: http://atrf.info/papers/2010/2010_Wang_Charles.pdf [Último acceso: 1/04/2018]. 